\title{
Fully Truncated Cone-Beam Reconstruction on Pi Lines Using Prior $\mathrm{CT}^{\star}$
}

\author{
Krishnakumar Ramamurthi ${ }^{1}$, Norbert Strobel ${ }^{2}$, \\ Rebecca Fahrig ${ }^{3}$, and Jerry L. Prince ${ }^{1}$ \\ 1 Johns Hopkins University, Electrical and Computer Engineering, \\ Baltimore, MD 21218, USA \\ krishna@jhu.edu, prince@jhu.edu \\ 2 Siemens Medical Solutions, Stanford University Medical Center, \\ Stanford, CA 94305, USA \\ Norbert.Strobel@siemens.com \\ 3 Stanford University, Department of Radiology, \\ Stanford, CA 94305, USA \\ fahrig@stanford.edu
}

\begin{abstract}
C-arms are well suited for obtaining cone-beam projections intra-operatively. Due to the compact size of the detector used, the data are usually truncated within the field of view. As a result, direct application of a standard cone-beam reconstruction algorithm gives rise to undesirable artifacts and incorrect values in the reconstructed image volume. When prior information such as a pre-operative CT scan is available, fully truncated cone-beam projections can be used to recover the change within a small region of interest without such artifacts. A method for integrating prior CT is developed using the concept of pi-lines and tested on real flat-panel and simulated cone-beam data.
\end{abstract}

\section{Introduction}

Three dimensional image reconstruction from C-arm cone-beam projections has been an area of growing practical interest over the last couple of decades. Carms can provide rotational cone-beam images that can be used for the purpose of three dimensional reconstruction of anatomy. Filtered backprojection methods, such as Feldkamp's method [1], are among the most popular approaches to cone-beam reconstruction of $\mathrm{C}$-arm images. These methods require a sufficiently large angular sweep, and require that the anatomy being scanned is entirely captured in the detector. In intra-operative applications, such as in hip replacement surgeries, these conditions cannot be practically met, due to patient size, orientation, and other objects such as tools, patient table, etc. that occlude the C-arm's view of the anatomy. These restrictions cause undesirable artifacts within the reconstruction field of view (FOV) thus rendering the reconstructions unsuitable for prognosis or planning. While intra-operative CT scans are quite impractical

\footnotetext{
* This work is jointly supported by NIH research grants R21-EB003616 (Johns Hopkins University), EB003524 (Stanford University) and Siemens Corporate Research.
} 
for such applications, prior CT scans of the same patient may provide valuable information that can be used to compensate for aforesaid artifacts.

In this paper, we investigate the possibility of using prior $\mathrm{CT}$ information in cone-beam reconstruction, from fully truncated C-arm images. Recent developments in fan-beam and cone-beam tomography [2, 3, 4, 5, have shown, by introducing the concept of "Pi-lines", that a certain degree of truncation (along the width of the detector) is admissible. However, when data is truncated at both ends of the detector these methods do not work. This is known as the interior problem and it has been shown that a unique inverse does not exist [6] Our goal is to show that in certain cases prior CT information can be used to convert the interior problem into a sub-interior problem, i.e., a case where the aforementioned $\mathrm{Pi}$-lines technique can be used. Consider for example an application involving the imaging of the pelvic bone during a sacroplasty procedure. Although a 3D pre-operative CT is typically obtained to find the regions of sacral fracture, it would also be helpful to obtain $3 \mathrm{D}$ reconstructions during the various steps of the procedure itself. We approach this problem by modeling the effects of the procedure as a change in the anatomy with respect to the prior CT. We show how this change can be recovered within a small region of interest from a semicircular sweep of heavily truncated cone-beam projections. The x-ray source can be collimated to as narrow a beam as required to cover this region of interest thereby keeping radiational exposure to a minimum.

\section{Background}

\subsection{Classical Framework}

Cone-beam reconstruction techniques may be broadly classified based upon the geometry of the source path: (a) Non-planar source paths and (b) Planar source paths. Exact 3D reconstruction is possible only when the source path satisfies Tuy's [7] condition, according to which every plane passing through any point of interest must intersect the source path non-tangentially at least once. It immediately follows that, only non-planar source paths are capable of satisfying this requirement for an entire volumetric region. Planar source paths can at best satisfy this requirement only for points contained within the plane containing the source path itself. In such cases it is simplest to develop cone-beam algorithms by simply extending existing exact $2 \mathrm{D}$ fan-beam reconstruction techniques above and below the mid-plane (the plane containing the source path). Feldkamp's reconstruction method [1] for volumetric cone-beam reconstruction is an example of this approach. It is an extension of classical exact $2 \mathrm{D}$ fan-beam reconstruction algorithm on a circular path.

\subsection{Recent Developments}

In recent years, exact fan-beam reconstruction techniques have improved progressively by relaxing the requirements on the projection data. While classical fan-beam tomography cannot handle truncated data and requires a minimum 
sweep of $\pi+$ fan-angle (short-scan), Noo et al [8] proposed a method that could work with a very short-scan sweep (less than a short-scan) for exact reconstruction in a region of interest (ROI) within the field of view (FOV). Truncation is still not admissible in this method however. An extension of this method to cone-beam tomography was proposed in 9, 10. An important breakthrough in handling data truncation in fan-beam tomography was presented in [1] by introducing the idea of a virtual fan-beam re-binning technique. An alternate and emerging approach for handling data truncation is to reconstruct the Hilbert transform of the underlying function on pi lines [2,3] by backprojecting the first derivative of the projection data. The Hilbert transform is then inverted on these lines to obtain the final reconstruction. These methods were largely inspired by a method introduced in helical CT by Zou et al in 2004 [12. The idea of pilines has also been generalized for the purpose of cone-beam reconstruction from arbitrary source paths [4,5].

\section{PI Lines Theory}

For completeness, since this is a relatively new concept, we now briefly describe the idea of cone-beam inversion on pi-lines [4]. For mathematical ease, we use the notion of a virtual detector centered at the origin $\mathbf{O}$ (see Fig. 11). The orthonormal vectors $\hat{\mathbf{u}}, \hat{\mathbf{v}}$ and $\hat{\mathbf{w}}$ define the orientation of the detector width, detector length, and the position of the x-ray source $\mathbf{a}(\lambda)$. The cone-beam projection data is given by a scalar function $g(u, v, \lambda)$. The x-ray source assumes various positions along its trajectory that is parameterized by $\lambda \in \Lambda$, a real interval. Without loss of generality it is assumed that the detector is well oriented along these various poses, i.e., $\mathbf{a}^{\prime}(\lambda)$ the tangent to the source trajectory is always parallel to $\hat{\mathbf{u}}$. A pi-line is defined as any line that connects two points of a connected source trajectory. Figure 1 shows a pi-line $\Pi_{\lambda^{*} \lambda}$ that contains $\mathbf{a}(\lambda)$ and $\mathbf{a}\left(\lambda^{*}\right)$. The only pi-lines that concerns us are those that intersect the virtual detector, since these are ones that are actually measured. Consider a point $\mathbf{x} \in \Pi_{\lambda^{*} \lambda}$ and let the underlying $\mathbf{x}$-ray attenuation function at this point be denoted as $f(\mathbf{x})$. Since $\Pi_{\lambda^{*} \lambda}$ is a measured line, it is safe to assume that $f(\mathbf{x})$ is of finite support say

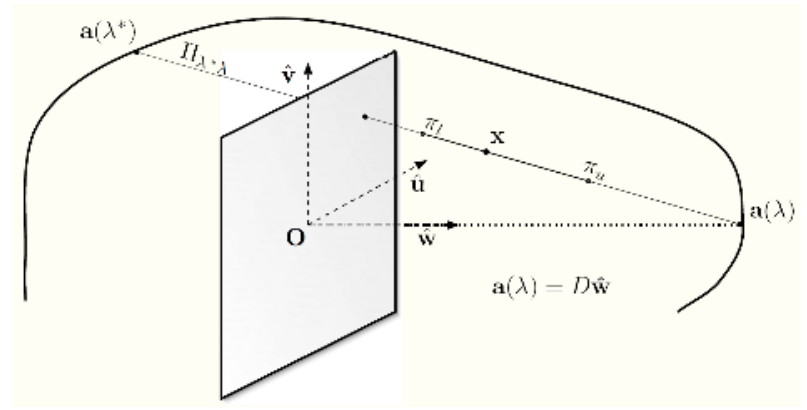

Fig. 1. Differentiated back projection in cone-beam geometry 
$\left(\pi_{l}, \pi_{u}\right)$, on this line. Although truncation is admissible in general, it is strictly required that this section of the pi-line $\Pi_{\lambda^{*} \lambda}$ be captured completely in all the views from $\lambda$ to $\lambda^{*}$. Under these assumptions it has been shown in [4] that the Hilbert transform of $f(\mathbf{x})$ along $\Pi_{\lambda^{*} \lambda}$ can be measured as follows:

$$
\begin{aligned}
\mathcal{H}_{\lambda^{*} \lambda}[f(\mathbf{x})]=\frac{1}{2 \pi} & {\left[\int_{\lambda}^{\lambda^{*}} \frac{D\left\|\mathbf{a}^{\prime}(\lambda)\right\| g_{F}\left(u^{*}(\lambda, \mathbf{x}), v^{*}(\lambda, \mathbf{x}), \lambda\right)}{[(\mathbf{a}(\lambda)-\mathbf{x}) \cdot \hat{\mathbf{w}}]^{2}} d \lambda\right.} \\
& \left.+\left.\frac{g\left(u^{*}(\lambda, \mathbf{x}), v^{*}(\lambda, \mathbf{x}), \lambda\right)}{\|\mathbf{a}(\lambda)-\mathbf{x}\|}\right|_{\lambda} ^{\lambda^{*}}\right],
\end{aligned}
$$

where $\left(u^{*}(\lambda, \mathbf{x}), v^{*}(\lambda, \mathbf{x})\right)$ is the point where $\Pi_{\lambda^{*} \lambda}$ intersects the virtual detector, and

$$
g_{F}(u, v, \lambda)=\frac{\partial}{\partial u}\left[\frac{D}{\sqrt{u^{2}+v^{2}+D^{2}}} g(u, v, \lambda)\right] .
$$

The Hilbert transform obtained in (1) is directed from $\mathbf{a}\left(\lambda^{*}\right)$ to $\mathbf{a}(\lambda)$. Let $t$ denote a scalar that specifies position on $\Pi_{\lambda^{*} \lambda}$ along this direction. A complete measure of $\mathcal{H}_{\lambda^{*} \lambda}[f(t)] \forall t \in\left(\pi_{l}, \pi_{u}\right)$ can then be obtained. Since the Hilbert transform is shift-invariant, any particular choice for $t=0$ will do. Under the condition that $f(t)$ vanishes outside the interval $\left(\pi_{l}, \pi_{u}\right)$, the Hilbert transform can be inverted by,

$$
f(t)=\frac{-1}{\sqrt{\left(t-\pi_{l}\right)\left(\pi_{u}-t\right)}} \int_{\pi_{l}}^{\pi_{u}} \frac{\sqrt{\left(t^{\prime}-\pi_{l}\right)\left(\pi_{u}-t^{\prime}\right)} \mathcal{H}\left[f\left(t^{\prime}\right)\right]}{\pi\left(t-t^{\prime}\right)} d t^{\prime}+C,
$$

where $C$ is a constant of integration that can be determined by the knowledge that $f(t)=0$ outside this interval.

\section{Methods}

In this section we present the two main contributions of this paper. We first show how to obtain the Hilbert transform on a set of parallel pi-lines from a set of diverging fan-beam (or cone-beam in the mid-plane) projections. We then show how to integrate prior information along these pi-lines from a prior CT. Since the source geometry is planar the method is approximate above and below the mid-plane. For explanatory purposes we use the "Popeye" phantom (see Fig. 2a) defined in [3] to serve as the prior $\mathrm{CT}\left(f_{p}(\mathbf{x})\right)$. A modified Popeye phantom $\left(f_{m}(\mathbf{x})\right.$ is then obtained by adding a long cylinder, and an ellipsoid, changes that are assumed to occur during surgery (Fig. 2b). The goal is to image a small ROI of this modified phantom using fully truncated cone-beam projections obtained over a semi-circular source path.

\subsection{Selective Differentiated Back-Projection}

As shown in Fig. 2b, the size of the detector is clearly too small to capture the modified Popeye phantom from any view angle $(g(u, v, \lambda)$ is only known $\forall u \in$ 


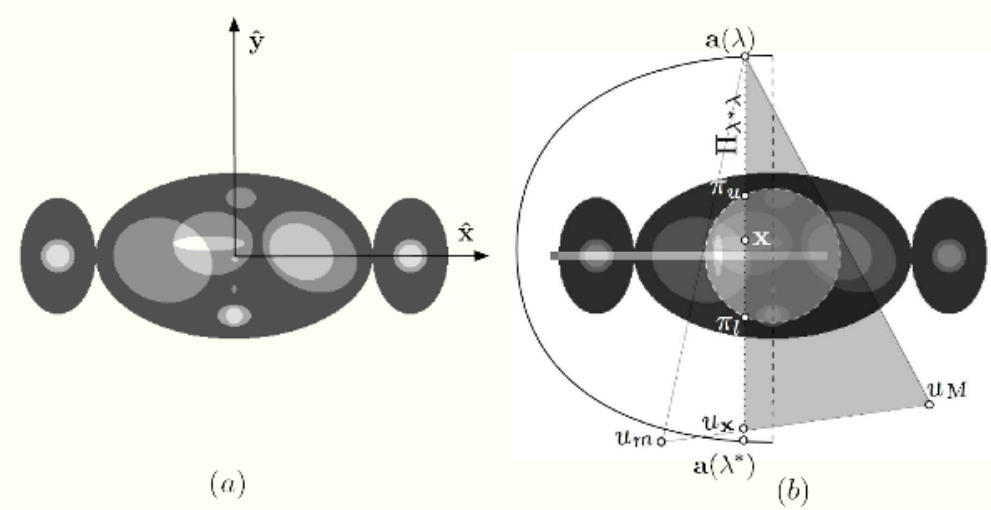

Fig. 2. (a) Popeye phantom serves as prior CT, (b) Selective backprojection to obtain Hilbert transform on parallel lines of modified Popeye phantom

$\left.\left[u_{m}, u_{M}\right]\right)$. However, the local nature of the derivative operation in (2) allows for accurate calculation of $g_{F}(u, v, \lambda) \forall u \in\left(u_{m}, u_{M}\right)$. Since the limits of the integral in (11) are dependent on the reconstruction point $\mathbf{x}$, a voxel-driven backprojection summation algorithm cannot be directly used. In order to get over this hurdle, we perform a selective differentiated backprojection of the data by defining a modified data function defined as follows:

$$
\tilde{g}_{F}(u, v, \lambda)=g_{F}(u, v, \lambda) \forall \quad u \quad \underset{u_{\mathbf{x}}>0}{\gtrless} u_{\mathbf{x}} .
$$

where $u_{\mathbf{x}}$ corresponds to the column on the detector where the vertical pi-line $\Pi_{\lambda^{*} \lambda}$ (see Fig. 2b) containing $\mathbf{a}(\lambda), \mathbf{x}$, and $\mathbf{a}\left(\lambda^{*}\right)$ intersects it. We backproject this modified projection data over the entire semi-circle to obtain

$$
\hat{\mathcal{H}}_{y}\left[f_{m}(\mathbf{x})\right]=\frac{1}{2 \pi} \int_{0}^{\pi} \frac{D\left\|\mathbf{a}^{\prime}(\lambda)\right\| \tilde{g}_{F}\left(u^{*}(\lambda, \mathbf{x}), v^{*}(\lambda, \mathbf{x}), \lambda\right)}{[(\mathbf{a}(\lambda)-\mathbf{x}) \cdot \hat{\mathbf{w}}]^{2}} d \lambda .
$$

Since the limits of the integration in (5) are not position dependent we can process the data using a typical cone-beam backprojection summation algorithm. The bounds in (4) ensure that for points lying on vertical pi-lines, backprojection is effectively computed only over the range defined by the intersection of such a pi-line with the source path. We have also ignored the second term of (1) arguing that its contribution to the summation is significantly less than that from the first term.

\subsection{Integration of Prior CT}

The selective backprojection of data on vertical pi-lines gives us a good estimate of the Hilbert transform of $f_{m}(\mathbf{x})$ along the positive $y$ axis. With the knowledge of 
$f_{p}(\mathbf{x})$ along the same pi-lines we can obtain an estimate of the Hilbert transform of the change $c(\mathbf{x})=f_{m}(\mathbf{x})-f_{p}(\mathbf{x})$ using the fact that the Hilbert transform is a linear transform, i.e.,

$$
\hat{\mathcal{H}}_{y}[c(\mathbf{x})]=\hat{\mathcal{H}}_{y}\left[f_{m}(\mathbf{x})\right]-\mathcal{H}_{y}\left[f_{p}(\mathbf{x})\right],
$$

where

$$
\mathcal{H}_{y}\left[f_{p}(\mathbf{x})\right]=\mathcal{H}_{y}\left[f_{p}(x, y, z)\right]=\int_{-\infty}^{+\infty} \frac{f_{p}\left(x, y^{\prime}, z\right)}{\pi\left(y-y^{\prime}\right)} d y^{\prime} .
$$

An estimate of $c(\mathbf{x})$ can then be evaluated by using (3) to invert $\hat{\mathcal{H}}_{y}[c(\mathbf{x})]$ over the domain $\left(\pi_{l}, \pi_{u}\right)$. This inversion is done over the entire FOV, and then added back to the prior CT to obtain our final reconstruction result. This method works well when $c(\mathbf{x})=0$ outside the FOV covered by the fully truncated scan. In this case since the change is a long object oriented along the $x$ axis, it makes sense to reconstruct along vertical pi-lines. It general it would be effective to perform the selective backprojection such that the pi-lines are oriented orthogonal to the longer dimension of the change.

\section{Results and Discussion}

We conducted two sets of experiments to test the method described in Sect. 4 Using the Popeye phantom and its modified version we began with a simulated experiment. We also conducted a real experiment using an anthropomorphic hip section phantom known as the Alderson Rando Phantom. We first obtained a complete CT scan of the entire hip phantom to serve as the prior CT for our experiment. The phantom consists of several slabs $(\approx 1 \mathrm{inch})$ that are stacked vertically. This allowed for easy modification of the interior section of the phantom. We drilled out 7 cylindrical plugs of varying diameters (3 to $7 \mathrm{~mm}$ ) from the most central slab (also coinciding with the mid-plane of the sweep). We then replaced three of these with Aluminum plugs. Using a Siemens flat panel ceiling mounted C-arm (Artis dTA), we then obtained well calibrated cone-beam projections of this modified hip phantom over a semi-circular sweep. The poses of the C-arm were accurately represented using projection matrices obtained by scanning an offline calibration phantom. For the purpose of registration we used a standard Levinson-Marquardt optimization routine that searched through a 6 parameter space to find a rigid body transformation that would take the prior $\mathrm{CT}$ to the space of the $\mathrm{C}$-arm projections. The cost function was the combined error in the prediction of where some known external markers would be projected to on the image domain. We used two orthogonal views for this purpose. The scan parameters for the two experiments are summarized in Table 1.

The results from the two experiments are shown in Fig. 3. In the case of the real data experiment, it was sufficient to apply our method on pi-lines that spanned only half of the FOV since there was no change outside this region. The results are shown on slices that correspond to the mid-plane of the source geometry. Figures $[3$ and 3 d show instances of the prior CT in each case. For the 
Table 1. Scan Parameters

\begin{tabular}{|l||c|c|}
\hline \multicolumn{1}{|c||}{} & Simulated Data & Real Data \\
\hline \hline Angular Range & $180^{\circ}$ & $180^{\circ}$ \\
\hline Angular Sampling & $1^{\circ}$ & $0.4^{\circ}$ \\
\hline Source to Detector & $1040 \mathrm{~mm}$ & $1180 \mathrm{~mm}$ \\
\hline Source to Iso-center & $570 \mathrm{~mm}$ & $785 \mathrm{~mm}$ \\
\hline Number of Rows & 256 & 1024 \\
\hline Number of Columns & 256 & 756 \\
\hline Row Spacing & $2.554 \mathrm{~mm}$ & $0.373 \mathrm{~mm}$ \\
\hline Col Spacing & $1.4 \mathrm{~mm}$ & $0.373 \mathrm{~mm}$ \\
\hline Prior CT grid & $(512,512,200)$ & $(512,512,200)$ \\
\hline Prior CT size & $(640 \mathrm{~mm}, 640 \mathrm{~mm}, 250 \mathrm{~mm})$ & $(380 \mathrm{~mm}, 380 \mathrm{~mm}, 200 \mathrm{~mm})$ \\
\hline \hline
\end{tabular}

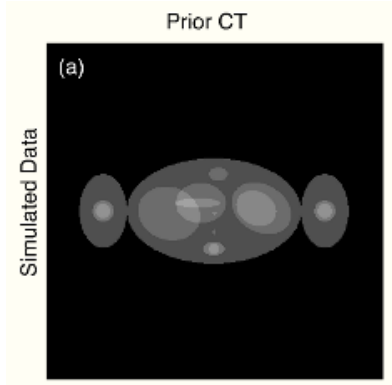

FDK versus priorCT

Our Method
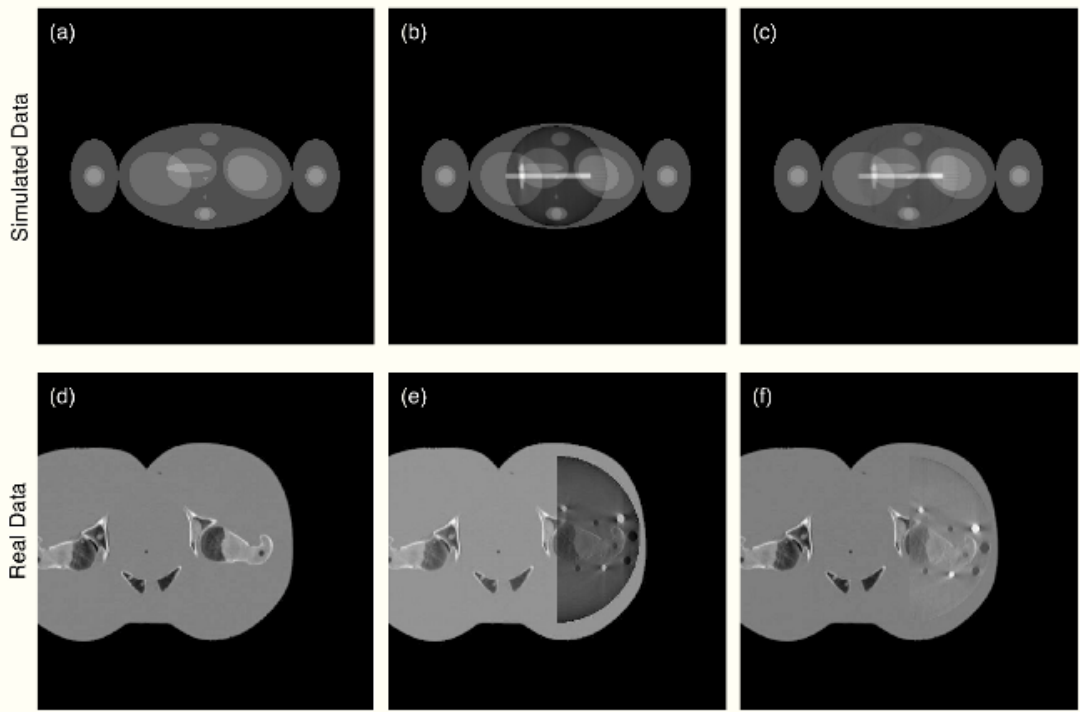

Fig. 3. Integration of prior $\mathrm{CT}$ information along pi-lines reduces truncation artifacts to a great extent

purpose of comparison, we show the results of applying the very short-scan FBP algorithm on fully truncated projections. We juxtapose these results with the prior CT outside the FOV in order to emphasize the mismatch in reconstructed $\mathrm{CT}$ values (see Figs. 3 $\mathrm{b}$ and 3e). The discrepancy in the values is not due solely to a DC offset but also to an indeterminable cupping attenuation that is typical of the interior problem. This is mainly an effect of the non-local nature of the ramp-filter that is used in such methods. The characteristic strong edge on the boundary of the FOV has been avoided by artificially setting the boundary value of the ramp-filtered projections to zero. Using our method instead, we present the 
results of adding the recovered change within the FOV to the prior CT. As can be seen in Figs. 3r. and 3f, our method does a remarkably good job of consistently fusing the two reconstructions. The improvement in the reconstructed values of our method is a result of mathematically consistent compensation along pi-lines, and not due to an ad-hoc scaling of values. In the case of real data however, we had to determine a scalar scale in order to match the C-arm data to the prior CT data. This was automatically done by comparing the average values of $\mathcal{H}_{y}\left[f_{p}(\mathbf{x})\right]$ and $\hat{\mathcal{H}}_{y}\left[f_{m}(\mathbf{x})\right]$ within the FOV.

\section{References}

1. L.A. Feldkamp, L.C. Davis, and J.W. Kress. Practical cone-beam algorithm. Optical Society of America, 1(6), June 1984.

2. Y. Zou, X. Pan, and E. Y. Sidky. Image reconstruction in regions-of-interest from truncated projections in a reduced fan-beam scan. Physics in Medicine and Biology, 50:13-27, DEC 2004.

3. F. Noo, R. Clackdoyle, and J. D. Pack. A two-step hilbert transform method for 2D image reconstruction. Physics in Medicine and Biology, 49:3903-3923, AUG 2004.

4. J. D. Pack, F. Noo, and R. Clackdoyle. Cone-beam reconstruction using the backprojection of locally filtered projections. IEEE Transactions on Medical Imaging, 24(1):70-85, JAN 2005.

5. T. Zhuang, S. Leng, B. E. Nett, and Chen G. Fan-beam and cone-beam image reconstruction via filtering the backprojection image of differentiated projection data. Physics in Medicine and Biology, 49:5489-5503, DEC 2004.

6. F. Natterer. The mathematics of computerized tomography. Wiley, New York, 1986.

7. H.K. Tuy. An inversion formula for cone-beam reconstruction. SIAM Journal of Applied Mathematics, 43:546-552, 1983.

8. Frederic Noo, Michel Defrise, Rolf Clackdoyle, and Hiroyuki Kudo. Image reconstruction from fan-beam projections on less than a short-scan. Physics in Medicine and Biology, 47:2525-2546, July 2002.

9. K. Ramamurthi and J. Prince. Tomographic reconstruction for truncated cone beam data using prior CT information. Sixth International Conference: Medical Imaging Computing and Computer-Assisted Intervention (MICCAI), pages 134$141,2003$.

10. F. Noo and H. D.J. Image reconstruction from cone-beam data on a circular short-scan. SPIE Medical Imaging, February 2002.

11. R. Clackdoyle, F. Noo, J. Guo, and J. A. Roberts. Quantitative reconstruction from truncated projections in classical tomography. IEEE Transactions on Nuclear Science, 51(5):2570-2578, OCT 2004.

12. Y. Zou and X. Pan. Exact image reconsturcion on PI-lines from minumum data in helical cone-beam CT. Physics in Medicine and Biology, 49:941-959, FEB 2004. 